\title{
Risk finance
}

Recurrent crises, the growth and awareness of complexity and unpredictable events, financial globalization and regulation have reaffirmed the limits of financial risk models that account for calculated and predicted risks and the importance of framing uncertainty into a mold we can better comprehend and manage. These have challenged risk finance from both theoretical and practical viewpoints. From a theoretical viewpoint, a number of issues have challenged fundamental finance, emphasizing the mis-match of micro and macro-economic factors in pricing financial assets, incomplete state preferences, global and strategic financial markets and not least the recurrence of "rare events", jump processes, stochastic volatility etc. that are rendering the "theory and the business" of risk finance far more difficult. For example, a distinction between what we mean by "predictability" or a lack of it is still a debated question. Is unpredictability embedded in randomness? Is unpredictability embedded in our lack of understanding, in an over-simplification of intricate relationships, their complexity and dependencies that beset us? Is unpredictability embedded in rare events or Black Swans? Is unpredictability embedded in the strategic encounters of parties with broadly varying agendas, information and power and their asymmetries? While in fact risk models are based on predictability, most of the issues challenging risk finance are those defined by risks that are not accounted for.

For example, theorists, such as Minsky, 1993, hypothesized that financial markets are regime-unstable (presented as an interpretation of the elements of Keynes' Theory of general equilibrium). In this framework, markets have financing regimes under which they are stable and others where they are not. In Minsky's theory there is a natural tendency for the economy to transit from a stable to an unstable system providing thereby a rationale for the booms and the busts (i.e. a dynamic equilibrium) that we often observe but have difficulty to explain. This inherent instability of financial markets is also difficult to reconcile with measurable risks and predictable outcomes.
In this vein, financial risk management calls for a greater appreciation of how to confront risk expost rather than just, ex-ante, once risks manifest themselves. Terms such "robustness", "resilience", "fragility" and "anti-fragility" are increasingly reappearing on the financial stage, recognizing the importance of both risk models (as the means to manage risk ex-ante) and alternative ex-post risk approaches (as mean to overcome financial consequences).

This special issue addresses specific and important risk problems.

The paper by Olivier Guéant, Tournament-induced risk-shifting: A mean field games approach, is based on a concept that reconcile (subject to some underlying assumptions) individual choices and their collective consequences. The Mean Field Games (MFG) approach provides an approximate solution to an infinite number of players game and therefore provides an approach to match micro-decisions and their macro consequences. In particular, Guéant considers an agency problem between an investor and his mutual funds managers and the implicit incentive induced by their compensation and risk shifting linked to a benchmark. Guéant's paper proposes a MFG model that quantifies the risk-shifting induced by a tournament-like competition between mutual funds.

Tyrone Carlin and Nigel Finch paper "Loan impairment provisioning under IAS 39 in the Asian banking sector" examine the response of a sample of Asian banks to the recognition of loan loss provision in the face of a gathering economic storm. Drawing on empirical data from 2006 through 2008, their paper focuses on the level of loan loss provisioning undertaken by the banks, with a view to generating insights into the effectiveness of the approach to loan impairment and provisioning prescribed by IAS 39 - Financial Instruments: Measurement and Recognition.

Fathi Abid, Mourad Mroua and Wing Keung Wong's paper consider whether Americans should invest internationally? To do so, they use both mean-variance portfolio optimization (MVPO) and stochastic domi- 
nance (SD) to investigate whether international diversification and home bias inertia are substitutes or complements for Americans investment strategies.

Srdjan D. Stojanovic's paper provides an approach to "Any-utility neutral and indifference pricing and hedging". To do so, the paper uses neutral and indifference pricing PDEs derived for general multidimensional Markovian diffusive market models with certain conditions imposed any utility of wealth.
Finally, Jia-Wen Gu, Wai-Ki Ching, Tak-Kuen Siu and Harry Zheng use a "Probabilistic Boolean network" (PBN) to model credit defaults. Such an approach is found to be particularly useful to model correlated defaults across different industrial sectors and business cycles. It also assesses the impact of business cycles on modeling and predicting correlated defaults. The paper uses real default data to build a PBN and explain the default structure and make reasonable predictions of joint default in different sectors. 